\title{
The adaptor protein CARD9 is required for innate immune responses to intracellular pathogens
}

\author{
Yen-Michael S Hsu' ${ }^{1}$, Yongliang Zhang ${ }^{2}$, Yun You ${ }^{1}$, Donghai Wang ${ }^{3}$, Hongxiu Li ${ }^{1}$, Omar Duramad ${ }^{2}$, \\ Xiao-Feng Qin ${ }^{2}$, Chen Dong ${ }^{2} \&$ Xin Lin ${ }^{1}$
}

The caspase-recruitment domain-containing adaptor protein CARD9 regulates the innate signaling responses to fungal infection. Here we show that CARD9 is required for innate immune responses against intracellular pathogens. We generated $\mathrm{Card}^{-1-}$ mice and found that CARD9-deficient macrophages had defects in activation of the kinases p38 and Jnk but not of transcription factor NF-kB after bacterial and viral infection. CARD9-deficient mice failed to clear infection and showed altered cytokine production after challenge with Listeria monocytogenes. In wild-type cells, we found CARD9 inducibly associated with both the intracellular 'biosensor' Nod2 and the serine-threonine kinase RICK. Our data demonstrate that CARD9 has a critical function in Nod2-mediated activation of p38 and Jnk in innate immune responses to intracellular pathogens.

The innate immune system uses pattern-recognition receptors, such as Toll-like receptors (TLRs) on the cell surface, to detect bacterial and viral infection ${ }^{1-3}$. Through various adaptor and/or scaffold molecules, TLRs induce multiple signaling cascades $^{3-9}$, leading to the activation of mitogen-activated protein kinases (MAPKs) and transcription factors, including NF-KB, AP-1 and IRF3, all of which can initiate inflammatory, antimicrobial responses ${ }^{10,11}$. Published studies have demonstrated the detailed biochemical mechanisms induced by various TLRs $^{1,3,11}$. However, emerging evidence indicates that other intracellular receptors and 'biosensors' are also important in innate immune responses elicited by intracellular microbial infection and activate multiple signaling cascades ${ }^{12,13}$.

The receptor Nod2 functions as an intracellular biosensor that recognizes myriad types of infectious bacteria ${ }^{12}$. After bacterial infection, antigen-presenting cells such as macrophages and dendritic cells engage and then phagocytose bacteria to neutralize infection. After uptake of the bacteria, antigen-presenting cells are activated by bacterial cell wall components such as lipopolysaccharide (LPS) of Gram-negative bacteria or peptidoglycan (PGN) of Gram-positive bacteria. In addition, muramyl dipeptide (MDP), a hydrolyzed byproduct of PGN produced after lysosomal digestion, and whole Listeria monocytogenes are potent activators of innate immunity mediated by Nod2 (refs. 13,14), which further associate with 'downstream' adaptor and effector molecules, leading to the activation of multiple signaling pathways ${ }^{12,15-21}$. Deletion and mutation analysis of the gene encoding Nod2 (Card15) has demonstrated that Nod2 is critical for innate immunity of the gastrointestinal lining and inflammatory responses. Although the molecular mechanism by which Nod2 activates NF- $\kappa B$ and IRF3 has been demonstrated ${ }^{12,15-20}$, the signaling components mediating Nod2-induced MAPK activation remain to be defined.

CARD9 is an adaptor molecule containing an $\mathrm{N}$-terminal caspaserecruitment domain (CARD) and a C-terminal coiled-coil domain ${ }^{22}$. Studies have shown that CARD9 expression is found mainly in lymphoid tissues and correlates with gastrointestinal lymphoid malignancy ${ }^{23}$, providing additional clues to the potential involvement of CARD9 in inflammation and immune activation. CARD9 has been suggested as a key adaptor in innate immune signaling by dectin-1dependent NF- $\mathrm{\kappa B}$ activation after fungal infection ${ }^{24}$. Although studies have shown that overexpression of CARD9 in HEK293 cells can activate NF- $\kappa B^{22}$, that NF- $\kappa B$ induction is very modest relative to the induction of other molecules involved in NF- $\mathrm{KB}$ activation, such as Bcl-10 and CARMA1 (ref. 25). Instead, overexpression of CARD9 strongly activates the kinases p38 and Jnk, both of which are pivotal in many immune responses ${ }^{26-28}$. To investigate that possible function of CARD9, we generated CARD9-deficient mice to determine the involvement of CARD9 in innate immune responses.

\section{RESULTS}

\section{Expression of Card9 and generation of Card9 $9^{-/-}$mice}

We first examined the expression pattern of Card9 in wild-type mice and found high expression in bone marrow, thymus, spleen and lung (a nonlymphoid organ enriched with effector cells for innate immune responses) but not lymph nodes (Fig. 1a). We also found no expression of Card9 in nonlymphoid organs such as brain, kidney and liver, consistent with previous findings ${ }^{22}$. Finally, we found high expression of Card 9 in the mouse macrophage cell line RAW264.7 (Fig. 1a). To further determine the subsets of cell types that

\footnotetext{
${ }^{1}$ Department of Molecular and Cellular Oncology and ${ }^{2}$ Department of Immunology, University of Texas, M.D. Anderson Cancer Center, Houston, Texas 77030, USA.

${ }^{3}$ The CBR Institute for Biomedical Research, Harvard Medical School, Boston, Massachusetts 02115, USA. Correspondence should be addressed to

X.L. (xllin@mdanderson.org).
} 
a
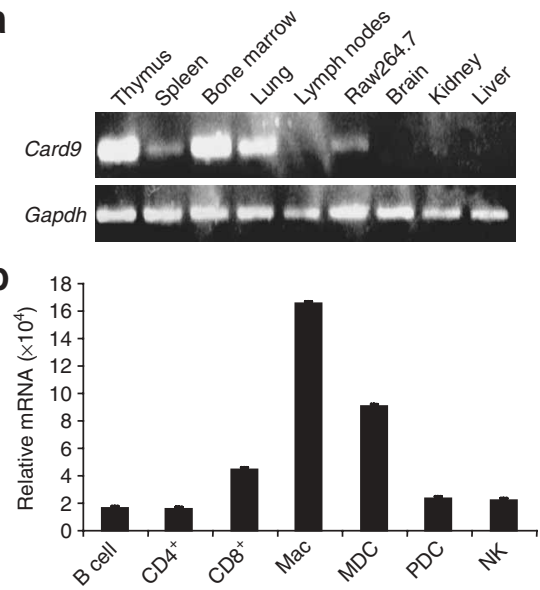

Figure 1 Characterization of the mouse Card9 expression profile. (a) RT-PCR of Card 9 mRNA expression, analyzed using total RNA extracted from various mouse organs and primers for exon 6 and exon 10 of the mouse Card9 coding sequence. Gapdh, loading control. (b) Real-time PCR analysis of Card9 expression in various lymphoid and myeloid cell types: $\mathrm{CD}^{+}$and $\mathrm{CD}^{+}$, T lymphocytes; Mac, macrophage; MDC, myeloid dendritic cell; PDC, plasmacytoid dendritic cell; NK, natural killer cell. Values are relative to Ubiquitin (Ubb1) expression. Results represent an average of three independent experiments (error bars, s.d.).

express Card9 in the immune system, we analyzed mRNA of various populations of immune cells by real-time PCR and found that Card9 was expressed mainly in macrophages and myeloid dendritic cells but not in T cells, B cells or natural killer cells (Fig. 1b), indicating that CARD9 is involved in innate immune responses.

To investigate the biological functions of CARD9, we used gene targeting to replace the first exon of mouse Card9, which contains the start codon as well as the coding region for the CARD, with a neomycin-resistance gene cassette driven by the promoter of the gene encoding phosphoglycerate kinase (Supplementary Fig. 1 online). We transfected the targeting vector into mouse embryonic stem cells by electroporation, selected neomycin-resistant cells and analyzed them by Southern blot for the targeted homologous recombination. We obtained a cell line with the targeted allele and used that to generate chimeric mice. The targeted allele successfully 'passed through' the germline to produce mice heterozygous for Card 9 deficiency (Supplementary Fig. 1). Mice homozygous for Card9 deficiency $\left(\mathrm{Card9}^{-1-}\right)$ were born at the normal mendelian ratio without obvious anatomical defects. Expression of CARD9 protein and the Card9 gene were completely abrogated in extracts from $\mathrm{Card}^{-1}$ primary macrophages and other tissues (Supplementary Figs. 1 and 2 online).
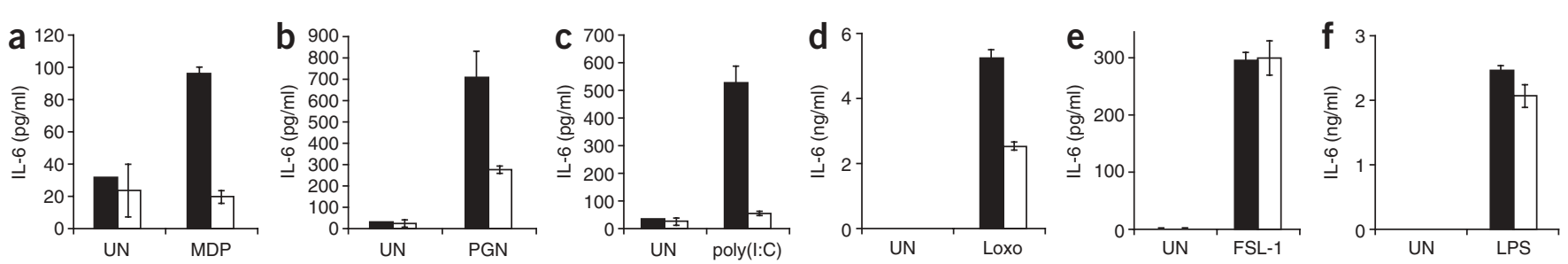

Figure 2 CARD9 is required for the production of proinflammatory cytokines after microbial mimetic stimulation. ELISA of IL-6 in the supernatants of wildtype (filled bars) and Card9 ${ }^{-1-}$ (open bars) peritoneal macrophages left unstimulated (UN) or stimulated for $24 \mathrm{~h}$ with MDP (10 $\mu \mathrm{g} / \mathrm{ml}$; a), PGN (10 $\mu \mathrm{g} / \mathrm{ml}$; b), poly(I:C) $(100 \mu \mathrm{g} / \mathrm{ml}$; c), loxoribine (Loxo; $1 \mathrm{mM}$; d), FSL-1 (1 ng/ml; e) or LPS (10 ng/ml; f). Results are representative of three to four independent experiments (error bars, \pm s.d. of triplicate experiments).

High expression of CARD9 in macrophages and myeloid dendritic cells but not lymphocytes suggested that CARD9 is functionally linked to the innate immune response. Consistent with that expression pattern, the development and activation of lymphocytes from Card $9^{-1-}$ mice were normal (data not shown). We therefore focused on determining the function of CARD9 in innate immune responses. We isolated primary macrophages from wild-type and $\mathrm{Card}^{-1-}$ mice and stimulated them with various synthetic microbial mimetics that elicit innate immune responses ${ }^{1}$. The production of proinflammatory cytokines interleukin 6 (IL-6) and tumor necrosis factor (TNF) by Card $9^{-1-}$ macrophages was defective after stimulation with poly(I:C) (a TLR3 ligand), loxoribine (a TLR7 ligand), PGN (a TLR2 ligand) or MDP (a Nod2 ligand; Fig. 2 and Supplementary Fig. 3 online). 'Dosage titration' of those agonists confirmed that CARD9 was required for innate immune-specific cytokine production (Supplementary Fig. 4 online). Unexpectedly, production of IL-6 and TNF induced by LPS (a TLR4 ligand) and diacylated lipopeptide FSL-1 (a TLR2 ligand) and production of TNF induced by PGN were not defective in Card $^{-1-}$ macrophages (Fig. 2 and Supplementary Figs. 3 and 5 online). These results collectively indicated that CARD9 selectively regulates innate immune responses.

\section{CARD9 regulates $\mathrm{p} 38$ but not NF-кB}

Because the IL-6 production defect was mostly noticeable in Card9-1macrophages treated with MDP and poly(I:C) (Fig. 2), we used those reagents to examine the biochemical mechanisms contributing to the cytokine production defect. In contrast to wild-type macrophages, Card $9^{-1-}$ macrophages failed to activate $\mathrm{p} 38$ in response to MDP and poly(I:C), whereas there was mild activation of the kinases Jnk and Erk in both wild-type and Card $9^{-/-}$macrophages (Fig. 3). Consistent with the LPS-induced cytokine production data (Fig. 2 and Supplementary Fig. 5), LPS-induced MAPK activation was normal in $\mathrm{Card9}^{-1}$ macrophages (Supplementary Fig. 6 online).

Because NF- $\kappa \mathrm{B}$ activation is an well established proinflammatory signaling pathway elicited by many pathogens ${ }^{9}$, we also examined the potential involvement of CARD9 in NF- $\kappa \mathrm{B}$ activation induced by MDP and poly(I:C). NF- $\kappa \mathrm{B}$ activation induced by MDP and poly(I:C) was normal in $\mathrm{Card9}^{-/}$cells (Fig. 3c,d), a result consistent with data showing that stimulation of $\mathrm{Card9}^{--}$cells by MDP or poly(I:C) induced degradation of $\mathrm{I} \kappa \mathrm{B} \alpha$ (Fig. 4a,b). These results suggested that CARD9 is selectively required for the activation of p38 but not of NF- $\kappa \mathrm{B}$ after stimulation of various innate immune receptors with bacterial and viral mimetics.

\section{CARD9 regulates intracellular innate immunity}

It is well documented that LPS and FSL-1 induce mainly TLRdependent signaling pathways, whereas poly(I:C), loxoribine and

\section{Responses to selective ligands require CARD9}




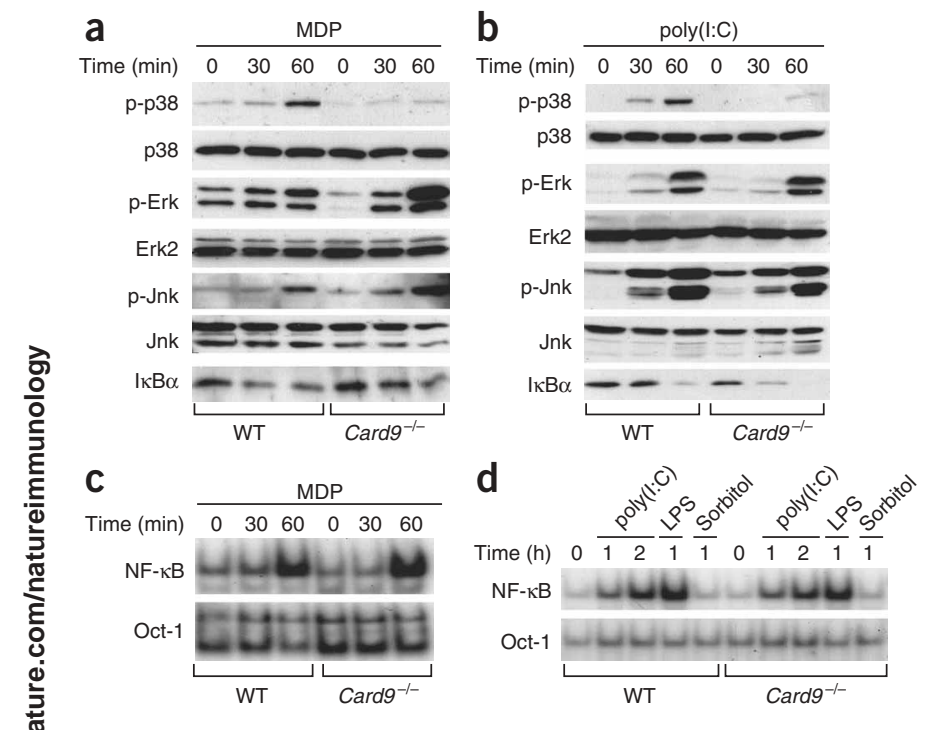

PGN can induce both TLR-dependent and TLR-independent signaling pathways ${ }^{14,29-31}$. In particular, MDP is a cleaved product of PGN that specifically activates Nod2 (ref. 14). Given the notable defects in MDPinduced signaling and cytokine production by $\operatorname{Card}^{-/}$cells, we speculated that the defects were mediated by a non-TLR pathway. Because MDP can 'bypass' the TLR system and specifically activates a TLR-independent signaling pathway ${ }^{3}$, we infected macrophages with the invasive bacterium $L$. monocytogenes to determine the involvement of CARD9 in bacteria-induced innate immune responses. L. monocytogenes, which normally activates Nod2-mediated inflammatory signaling pathways, failed to elicit full IL-6 and TNF production by Card $9^{-/-}$macrophages (Fig. 4a,b). Similarly, because poly(I:C) serves as a functional 'mimic' for double-stranded RNA viruses, we investigated the involvement of CARD9 in the RNA virus-induced innate immune response to vesicular stomatitis virus, which is known to activate TLR-independent antiviral signaling ${ }^{13}$. After infection with either vesicular stomatitis virus or lentivirus, we found a similar defect
Figure 3 Signaling defects of CARD9-deficient macrophages. $(\mathbf{a}, \mathbf{b})$ Immunoblot of MAPK phosphorylation and $I \kappa \mathrm{B} \alpha$ degradation in lysates of wild-type (WT) and $\mathrm{Card}^{-/-}$peritoneal macrophages stimulated (time, above lanes) with MDP (10 $\mu \mathrm{g} / \mathrm{ml}$; a) or poly(l:C) $(30 \mu \mathrm{g} / \mathrm{ml}$; b). p-, phosphorylated. (c,d) EMSA of NF-KB activation in nuclear extracts from wild-type or $\mathrm{Card}^{-/-}$macrophages stimulated (time, above lanes) with MDP $(10 \mu \mathrm{g} / \mathrm{ml} ; \mathbf{c})$ or with poly(l:C) $(100 \mu \mathrm{g} / \mathrm{ml})$, LPS $(100 \mathrm{ng} / \mathrm{ml})$ or sorbitol $(0.2 \mathrm{M}$; d). Oct-1, loading control. Results are representative of three independent experiments.

in proinflammatory cytokine production in $\mathrm{Card}^{-/-}$and wild-type macrophages (Supplementary Fig. 7 online). Collectively, these results indicated that CARD9 is critical in innate immune responses.

To provide the potential mechanism for the proinflammatory cytokine production defect, we examined activation of MAPK and NF- $\kappa \mathrm{B}$ in bacteria- and virus-infected $\mathrm{Card9}^{-1}$ macrophages. Although there was similar $\mathrm{I} \kappa \mathrm{B} \alpha$ degradation (Fig. 4c, bottom) and NF- $\kappa \mathrm{B}$ activation (Fig. 4d) in wild-type and Card9 $9^{-1}$ macrophages, p38 phosphorylation was much lower in the latter (Fig. 4c), consistent with the poly(I:C)- and MDP-induced p38 activation defect (Fig. 3). Also, activation of Jnk induced by bacterial and viral infection was lower in Card9 $^{-1-}$ macrophages (Fig. 4c), suggesting that CARD9 may also regulate Jnk activation. The results collectively suggested that CARD9 may function as an adaptor connecting intracellular bacterial and viral biosensors to the p38 and Jnk pathways. As it is well established that activation of p38 and Jnk are required for the production of proinflammatory cytokines ${ }^{26-28}$, the defect in the production of proinflammatory cytokines after intracellular bacterial and viral infection was probably due to the defect in the activation of p38 and Jnk.

\section{CARD9 physically associates with Nod2 and the kinase RICK}

Studies have shown that Nod2 functions as an intracellular bacterial biosensor. After bacterial infection, Nod2 uses its CARD motifs to associate with downstream CARD-containing proteins such as the serine-threonine kinase RICK, leading to activation of $\mathrm{NF}-\kappa^{12}$. However, the signaling components that mediate Nod2-induced MAPK activation have not been defined. Because Nod2 is the intracellular biosensor for $\mathrm{MDP}^{31}$ and CARD9 seems to regulate

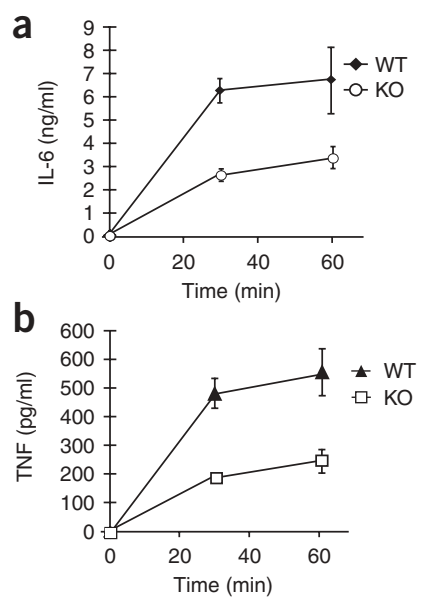

C

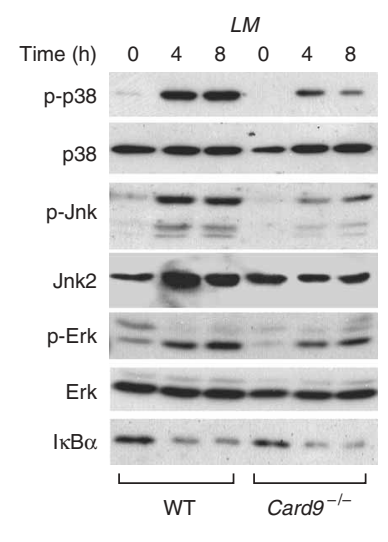

d

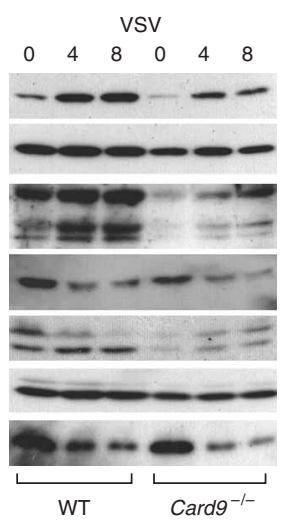

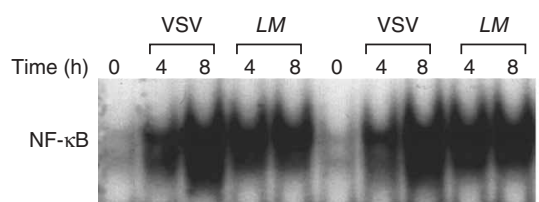

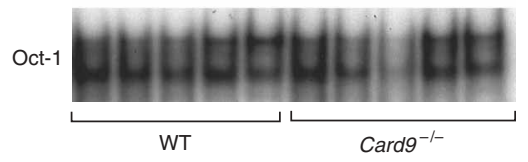

Figure 4 Induction of proinflammatory cytokines and activation of p38 and Jnk require CARD9 during intracellular bacterial and viral infection. (a,b) ELISA of IL-6 (a) and TNF (b) in supernatants from wild-type and Card9-- bone marrow-derived macrophages infected with $L$. monocytogenes (multiplicity of infection, 10). (c) Immunoblot analysis of MAPK phosphorylation and $1 \kappa B \alpha$ degradation in extracts of bone marrow-derived macrophages infected (time, above lanes) with L. monocytogenes ( $L M$; multiplicity of infection, 10 ) or vesicular stomatitis virus (VSV; multiplicity of infection, 3 ). (d) EMSA of NF- $\mathrm{B}$ activation in nuclear extracts of bone marrow-derived macrophages infected with L. monocytogenes or vesicular stomatitis virus. Oct-1, loading control. Results are representative of three independent experiments (error bars (a,b), \pm s.d. of triplicate experiments). 
a

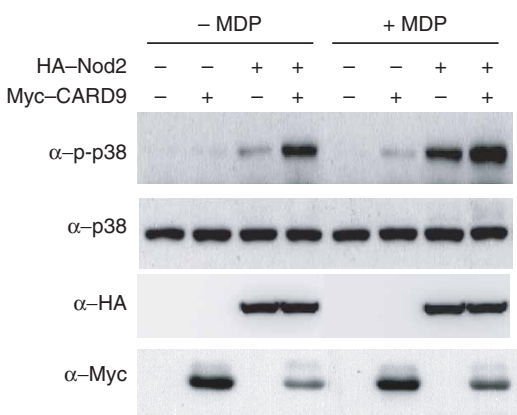

b

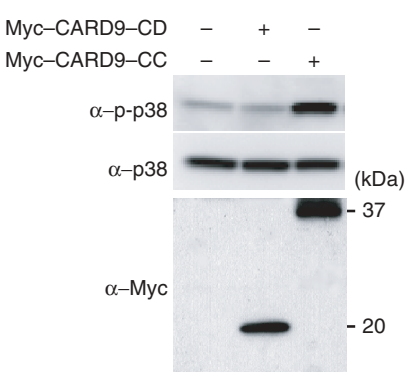

C

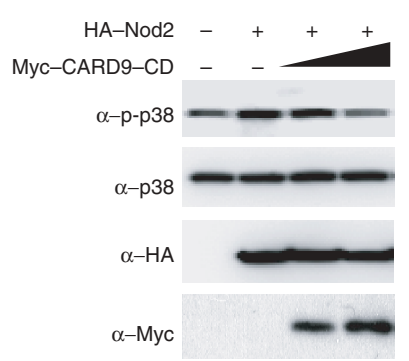

Figure 5 CARD9 acts in synergy with Nod2 in the activation of p38 but not of NF-kB. (a-c) Immunoblot analysis of lysates of HEK293T cells transiently expressing various proteins. (a) Cells expressing hemagglutinin-tagged Nod2 (HA-Nod2) and Myc-tagged CARD9 (Myc-CARD9) alone or in combination for $18 \mathrm{~h}$ and then left unstimulated or stimulated for $1 \mathrm{~h}$ with MDP (10 $\mu \mathrm{g} / \mathrm{ml})$. (b) Cells expressing Myc-tagged CARD (Myc-CARD9-CD) or coiled-coil domain (Myc-CARD9-CC) of human CARD9 for $18 \mathrm{~h}$. (c) Cells transfected for $18 \mathrm{~h}$ with plasmid expressing to. (d,e) Luciferase assay of the activation of ATF-2 and NF-KB in lysates of HEK293T cells transfected with plasmids expressing ATF-2-yeast transcriptional activator Gal4 and Gal4 promoter-luciferase (d) or NF-KB promoter-luciferase and EF1 $\alpha$ promoter-luciferase (e) plus plasmids expressing hemagglutinin-tagged Nod2 or Myc-tagged CARD9 or both. Relative ATF-2 or NF-kB activity is determined by normalization of the activity of ATF-2- or NF-kB-dependent firefly luciferase to that of renilla luciferase. Results are representative of two to three independent experiments.

p38 activation by MDP, we hypothesized that CARD9 functions downstream of Nod2. To investigate whether CARD9 functionally associates with Nod2, we expressed CARD9 with or without Nod2 in HEK293T cells. We found that whereas expression of either CARD9 or Nod2 activated both p38 and Jnk, expression of both CARD9 and Nod2 led to synergistic activation of p38 and Jnk (Fig. 5a and Supplementary Fig. 8 online). Stimulation of the transfected cells with MDP further enhanced CARD9- and NOD2-induced p38 activation (Fig. 5a), suggesting that CARD9 and Nod2 functionally cooperate in response to MDP stimulation.

To better understand the mechanism of CARD9-mediated p38 activation, we expressed deletion mutants of CARD9 in HEK293T cells. We found that expression of the coiled-coil domain but not the (2) CARD of CARD9 could activate p38 (Fig. 5b). Therefore, we thought that expression of the CARD alone might function as a dominant negative mutant for Nod2-induced p38 activation. Indeed, expression of the CARD itself inhibited Nod2-induced p38 activation (Fig. 5c). Consistent with the function of CARD9 in p38 activation, expression of CARD9 and Nod2 together synergistically induced the expression of a luciferase reporter under the control of ATF-2 (Fig. 5d), a transcription factor that functions downstream of $\mathrm{p} 38$. In contrast, expression of CARD9 did not affect Nod2-induced NF- $\mathrm{KB}$ activation (Fig. 5e). These results collectively indicated that CARD9 functions downstream of Nod2, leading to p38 activation.

Because both CARD9 and Nod2 contain CARDs, we next examined whether CARD9 physically associates with Nod2 to mediate p38 and Jnk activation. When they were expressed together, CARD9 specifically associated with Nod2 (Fig. 6a). To further assess its ability to interact with Nod2 in physiological conditions, we immunoprecipitated CARD9 from lysates of RAW264.7 macrophages infected with L. monocytogenes for various times. We detected a robust band of approximately 116 kilodaltons by immunoblot with antibody to Nod2 (anti-Nod2) in cellular lysates immunoprecipitated with anti-CARD9 at the 30-minute time point (Fig. 6b). To further verify the specificity of the interaction between endogenous CARD9 and Nod2 from primary cells, we used lysates of wild-type and CARD9-deficient a

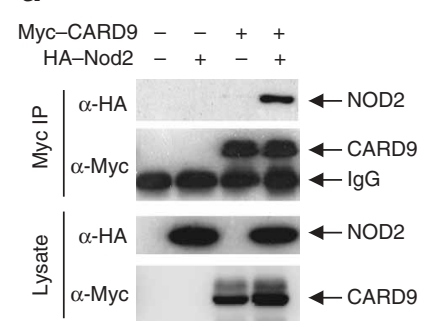

b

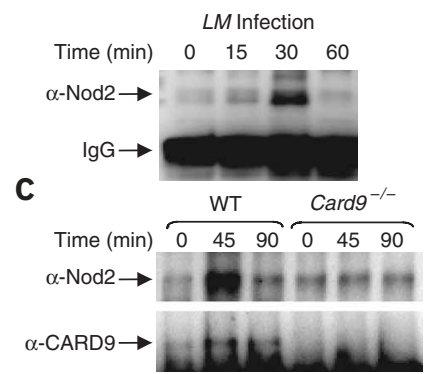

d

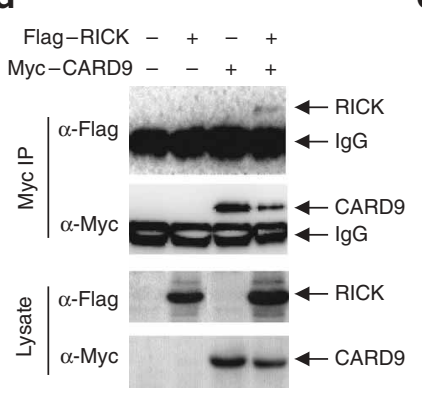

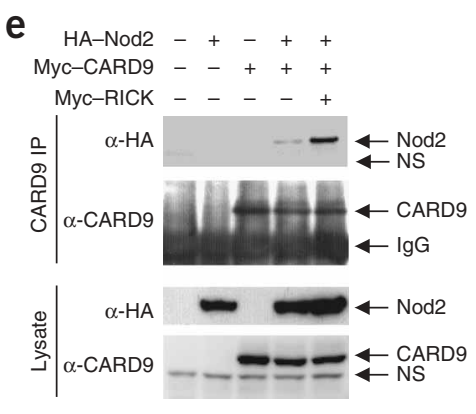

Figure 6 CARD9 associates with Nod2 in innate immune responses. (a) Immunoassay of HEK293T cells transiently transfected for $18 \mathrm{~h}$ with hemagglutinintagged Nod2 $(2 \mu \mathrm{g})$ with or without Myc-tagged CARD9 $(1 \mu \mathrm{g})$; lysates were immunoprecipitated (IP) with anti-Myc, then immunoprecipitates and lysates were analyzed. (b,c) Immunoassay of endogenous Nod2 association in RAW264.7 cells (b) or wild-type and Card $9^{-/-}$bone marrow-derived macrophages (c) infected with L. monocytogenes (time, above lanes); lysates were immunoprecipitated with anti-CARD9 and analyzed. IgG, immunoglobulin G. (d) Immunoassay of HEK293T cells transfected for $18 \mathrm{~h}$ with expression plasmids encoding Myc-tagged CARD9 and/or Flag-tagged RICK (Flag-RICK); lysates were immunoprecipitated with anti-Myc, then immunoprecipitates and lysates were analyzed. (e) Immunoassay of HEK293T cells transfected for $18 \mathrm{~h}$ with Myc-tagged CARD9, hemagglutinin-tagged Nod2 and/or Myc-tagged RICK (above lanes); lysates were immunoprecipitated with anti-CARD9, then immunoprecipitates and lysates were analyzed. NS, nonspecific. Results are representative of two to three independent experiments. 
a

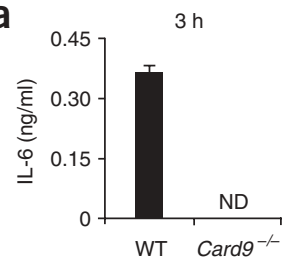

C

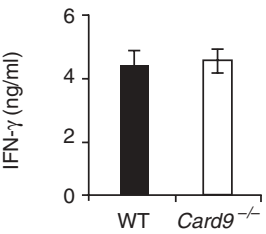

$24 \mathrm{~h}$
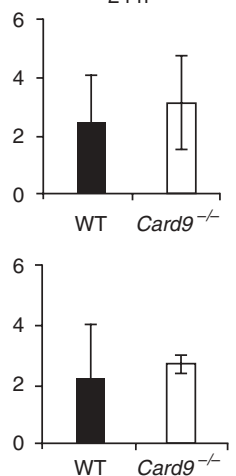

$48 \mathrm{~h}$
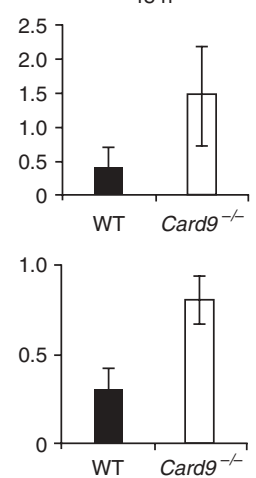
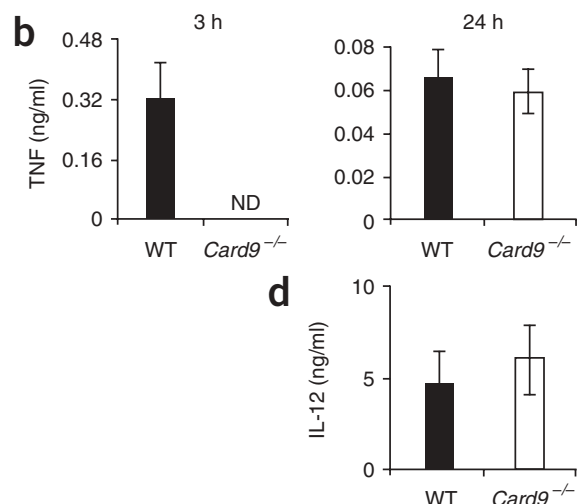

$48 \mathrm{~h}$
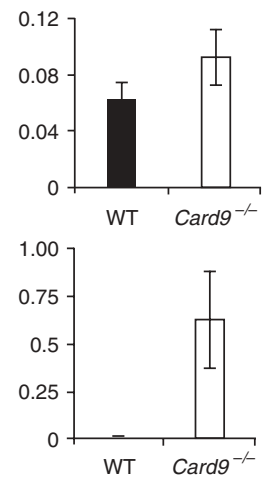

Figure 7 CARD9 is required for the early production of proinflammatory cytokines in response to intracellular bacteria infection. ELISA of IL-6 (a), TNF (b), interferon- $\gamma($ IFN- $\gamma ;(\mathbf{c}))$ and IL-12 (d) in sera from wild-type and Card9-/- mice infected with L. monocytogenes for 3,24 or 48 h. Results are representative of three independent experiments (error bars, s.d. derived from an average of three to four mice).

macrophages infected with $L$. monocytogenes for the immunoprecipitation. A robust protein band detected by immunoblot with antiNod2 was present at the 45-minute time point for wild-type but not in Card9 $9^{-1-}$ macrophages (Fig. 6c). Finally, because RICK has been shown to be critical in Nod2-mediated NF- $\kappa$ B activation downstream of Nod2 (ref. 19), we determined whether CARD9 associated with RICK. Although we found that RICK weakly associated with CARD9 when they were both overexpressed in HEK293 cells (Fig. 6d), the interaction between Nod2 and CARD9 was enhanced when RICK was coexpressed with Nod2 and CARD9 in HEK293 cells (Fig. 6e), suggesting that Nod2, RICK and CARD9 may form a functional complex. However, because we were unable to detect association of endogenous CARD9 with RICK (data not shown), a definite conclusion regarding the possible physical association of CARD9, Nod2 and RICK was not possible. Nevertheless, our results suggested that CARD9 may be a signaling component downstream of Nod2, leading to activation of MAPK but not of NF- $\kappa B$ (Supplementary Fig. 9 online).

\section{Card $^{-1-}$ mice fail to mount antibacterial immunity}

To investigate if CARD9 was required for immune responses to an intracellular pathogen in vivo, we administered a sublethal dose of L. monocytogenes to wild-type and $\operatorname{Card}^{-1-}$ mice by tail vein injection for various times. At $3 \mathrm{~h}$ after the injection, wild-type mice had higher production of IL-6 and TNF, the proinflammatory cytokine associated with early innate immune responses (Fig. 7a,b). In contrast, consistent with the essential function of CARD9 in MDPand PGN-induced cytokine production, serum IL-6 and TNF were not detectable in $\mathrm{Card}^{-1}$ mice (Fig. 7a,b), although there was no substantial difference in interferon- $\gamma$ production by wild-type and
Card9 $^{-1-}$ mice (Fig. 7c). In contrast, at 24 and $48 \mathrm{~h}$ after infection, systemic cytokines IL-6, TNF, interferon- $\gamma$ and IL-12 were all progressively higher in both wild-type and $\mathrm{Card}^{-1-}$ mice (Fig. 7c,d). Of note, the larger amounts of IL-6, interferon- $\gamma$ and IL-12 in Card $9^{-1-}$ mice were much greater than those in wild-type mice at $48 \mathrm{~h}$ after infection (Fig. 7), which might have been due to the failure in clearing the circulating bacteria.

As a possible explanation for the more abundant cytokines in the Card9 $^{-1-}$ mice, we examined the mice for signs of exacerbated of bacterial infection in their organs. Because the spleen and liver are the most commonly targeted organs during sepsis, we inspected those organs visually. As anticipated, we found noticeable spotty colonies of discoloration in $\operatorname{Card9}^{-1-}$ but not wild-type mice at $48 \mathrm{~h}$ to $9 \mathrm{~d}$ after infection (Supplementary Fig. 10 online). At day 9 after infection, there was considerable enlargement of the spleens from Card $^{-1-}$ mice (Supplementary Fig. 10). In the most severe cases, the spleens from $\mathrm{Card}^{-/-}$mice showed signs of necrosis (Supplementary Fig. 10). Further bacterial analysis at $2 \mathrm{~d}$ after infection showed that infected Card9 $^{-/}$mice had a bacterial burden in the liver and spleen more than 50- and 10-fold, respectively, that in wild-type mice (Fig. 8). The lack of bacterial clearance in Card9 $^{-1}$ mice continued on days 4 and 9 , whereas wild-type mice were capable of clearing most of the infection by day 4 (Fig. 8) and completely cleared the bacteria by day 9 (Fig. 8). These in vivo results demonstrated that CARD9 is essential for innate immune responses to intracellular bacteria.

\section{DISCUSSION}

Pattern-recognition receptors such as TLRs and other intracellular bacterial and viral receptors and biosensors induce innate immune
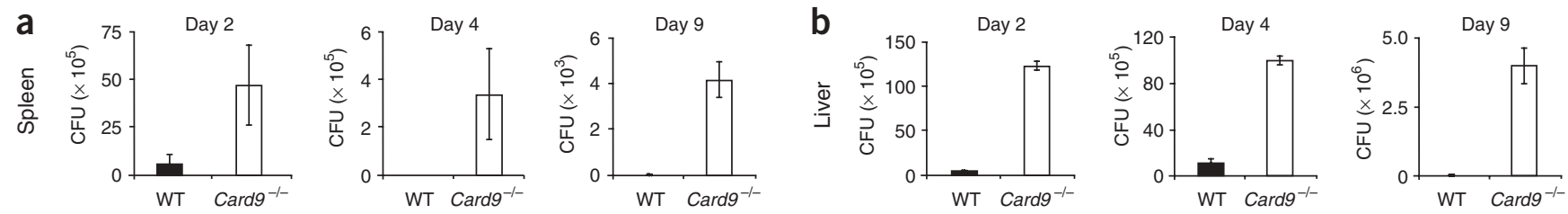

Figure $8 \mathrm{Card}^{-/-}$mice are impaired in the clearance of intracellular bacteria. Quantification of live L. monocytogenes in spleens (a) and livers (b) of wild-type and $\mathrm{Card}^{-/-}$mice at 2, 4 or $9 \mathrm{~d}$ after infection. Organs were homogenized and plated on listeria agar plates for determination of the growth of residual bacteria in colony-forming units (CFU). Results are representative of three independent experiments (error bars, s.d. derived from an average of three to four mice). 
responses after bacterial and viral infection. Various adaptor and/or scaffold molecules have important functions by 'connecting' the pattern-recognition receptors to downstream effector molecules, leading to the activation of multiple signaling pathways. By generating and characterizing CARD9-deficient mice, we have demonstrated here that CARD9 is a critical signaling component involved in innate immune responses to intracellular pathogens. Our results indicate that CARD9 is selectively involved in the activation of p38 and Jnk. It is well documented that activation of p38 and Jnk is required for the 할 production of proinflammatory cytokines ${ }^{26-28}$. Therefore, CARD9 deficiency results in an impaired production of proinflammatory cytokines after intracellular bacterial and viral infection.

Bacterial and viral infection induces both TLR-dependent and TLRindependent signaling pathways ${ }^{1,12,13}$. Among the various synthetic and purified TLR ligands, LPS and FSL-1 induce mainly TLR-dependent signaling pathways, whereas PGN, poly (I:C) and loxoribine can induce both TLR-dependent and TLR-independent signaling pathways ${ }^{14,29-31}$. In contrast, MDP activates only TLR-independent signaling pathways $^{14}$. The selective requirement of CARD9 in the response to poly (I:C), PGN, MDP and loxoribine but not to LPS and FSL-1 suggested that CARD9 participates in TLR-independent signaling pathways. Consistent with that possibility, we found that CARD9 was required for cytokine production in response to intracellular bacterial and viral infection. We also found a physical association of CARD9 with Nod2 ously expressed CARD9 and Nod2 after infection by L. monocytogenes, bacteria and bacteria and viruses. Although those data were clear, ultimately proof that CARD9 is selectively involved in only TLR-independent signaling by cells after exposure to pathogens will require the generation of mice lacking both CARD9 and specific TLRs. Nevertheless, our studies have demonstrated the requirement for CARD9 in specific signaling pathways and cytokine production in response to stimulation with selective bacterial and viral mimetics or bacterial and viral infection.

Consistent with the essential function of CARD9 in MDP- and PGN-induced cytokine production, the early production of proinflammatory cytokines in the sera of $\operatorname{Card9}^{-1}$ mice was defective at $3 \mathrm{~h}$ after L. monocytogenes infection. However, at later time points of infection (24 and $48 \mathrm{~h}$ ), Card $^{-1}$ mice had increased systemic proinflammatory cytokines. That phenomenon was probably due to a failure to clear the circulating bacteria, as demonstrated by the higher bacteria load found in spleens and livers from $\mathrm{Card}^{-1-}$ mice. Because CARD9 is expressed only in macrophages and dendritic cells, the higher cytokines detected at the late time point were probably due to cytokine production from other cells in response to circulating bacteria.

The in vivo phenotype of L. monocytogenes infection of Card9-/mice mimics the manifestation of clinical septic shock, and L. monocytogenes is one of the most common bacteria found in sepsis. During infection with bacteria, if the invading pathogen breaches the epithelium and enters the bloodstream, innate immune components will initiate a first 'wave' of immune responses to eliminate and arrest bacterial propagation in the liver and spleen. In actual sepsis, circulating bacteria are not cleared and instead they elicit soaring inflammatory reactions from immune effector cells such as natural killer cells and neutrophils. Because infected $\mathrm{Card9}^{-1}$ mice produce some of those same characteristics of sepsis after L. monocytogenes infection, Card $9^{-1-}$ mice may serve as a useful animal model for studying severe innate immune responses and sepsis.

Although overexpression of CARD9 can induce NF- $\kappa B$ activation ${ }^{22}$, $N F-\kappa B$ activation was relatively modest compared with that of other signaling components involved in NF- $\mathrm{KB}$ activation, suggesting that CARD9 is not involved in those signaling pathways that potently induce NF- $\kappa B$ activation. Consistent with that idea, we found that CARD9 deficiency did not affect the NF- $\kappa \mathrm{B}$ activation induced by TLRs and TNF (data not shown). In contrast to the modest induction of NF- $\mathrm{KB}$ in the previous study ${ }^{22}$, we found that overexpression of CARD9 potently induced activation of p38 and Jnk. Consistent with those results, purified microbial compounds such as MDP and poly(I:C) strongly activated p38 in wild-type but not $\mathrm{Card}^{-1-}$ macrophages, and activation of p38 and Jnk occurred after infection with either L. monocytogenes or vesicular stomatitis virus. Thus, we found that CARD9 is crucial for innate immune responses by regulating activation of p38 and Jnk after bacteria and viral infection. Because we found that CARD9 associates with Nod2, we propose that CARD9 may be the adaptor molecule connecting Nod2 to the downstream p38 and Jnk pathways. Studies identifying the downstream components of CARD9 will be essential for demonstrating the molecular mechanism by which p38 and Jnk are activated after bacterial infection.

A recent study suggests that CARD9 can mediate a non-TLR signaling pathway for antifungal immunity by mediating dectin-1induced NF- $\mathrm{KB}$ activation ${ }^{24}$. That study concluded that CARD9 is involved in a TLR-independent signaling pathway required for responses to fungal infection ${ }^{24}$. Because that study did not investigate the activation of p38 and Jnk induced by fungal infection or by stimulation with zymosan (a component of fungal cell walls), future studies should investigate whether CARD9-mediated p38 and Jnk activation is also involved in antifungal immunity. One notable difference between that study ${ }^{24}$ and our work reported here is that the previous study found normal antibacterial responses in the $\operatorname{Card~}^{-1-}$ mice that were produced and analyzed ${ }^{24}$. The difference between our data and those previous data may be due to use of different bacterial pathogens. The previous study used Staphylococcus aureus $^{24}$, which is known to induce TLR-dependent responses, whereas we used the intracellular bacterium L. monocytogenes, which potently induces TLR-independent responses. Additionally, in contrast to those previous findings showing that CARD9 and Bcl-10 synergistically activated NF- $\kappa B$ activation ${ }^{24}$, we found that CARD9 did not enhanced Bcl-10-induced NF- $\kappa \mathrm{B}$ activation. Nevertheless, both our study and that previous study ${ }^{24}$ have demonstrated essential functions for CARD9 in non-TLR-mediated innate immune responses elicited by microbial pathogens.

\section{METHODS}

Antibodies and plasmids. CARD9 polyclonal antibodies were produced by immunization of rabbits with a peptide 16 amino acids in length (DRGNTTGSDNTDTEGS) comprising residues 590-605 of CARD9. AntiMyc (A-14) (SC-789), anti-Nod2 (SC-30199) and anti-HA (SC-7392) were purchased from Santa Cruz Biotechnology. Anti-FLAG (F3165) was purchased from Sigma. Antibodies to p38 (9212), phosphorylated p38 (4631), phosphorylated Erk (9101), Jnk (9252) and phosphorylated Jnk (9251) were from Cell Signaling Technology. Myc-tagged CARD9 was constructed by cloning of human CARD9 cDNA into the pRK6-myc vector. Flag-tagged p38 and Flagtagged Bcl-10 were generated by cloning p38 and Bcl-10 cDNAs into the pCMV-Tag2B vector.

Cell sorting. Various lymphoid and myeloid cell types were sorted from the spleens of C57BL/6 mice. Cells were stained with combinations of fluorescence-conjugated monoclonal antibodies (BD Pharmingen or Miltenyi) and were sorted with a FACSAria (Becton Dickinson). The following sorting criteria were used for each cell type: $\mathrm{B}$ cell, $\mathrm{CD} 19^{+}$; $\mathrm{CD} 4 \mathrm{~T}$ cell, $\mathrm{CD} 3{ }^{+} \mathrm{CD} 4^{+}$; $\mathrm{CD} 8 \mathrm{~T}$ cell, $\mathrm{CD}^{+} \mathrm{CD}^{+}$; macrophage, $\mathrm{CD} 11 \mathrm{~b}^{+} \mathrm{F} 4 / 80^{+}$; myeloid dendritic cell, $\mathrm{CD} 11 \mathrm{c}^{+} \mathrm{CD} 11 \mathrm{~b}^{+}$; plasmacytoid dendritic cell, $\mathrm{CD} 11 \mathrm{c}^{+} \mathrm{B} 220^{+} \mathrm{mPDCA}^{+}$; natural 
killer cell, CD49b (DX5) ${ }^{+}$. The purity of all the sorted cell types was over $96 \%$, as determined by post-sorting flow cytometry on a FACSCalibur (Becton Dickinson).

RNA extraction and real-time PCR. For RT-PCR, total RNA was extracted with the RNeasy Mini kit (Qiagen) and reverse transcription was done with Superscript III (Invitrogen) to obtain cDNA. Primers specific for Il6, Tnf and glyceraldehyde phosphate dehydrogenase (Gapdh) were designed and purchased (Invitrogen; Supplementary Methods online). For real-time PCR, total RNA was extracted from sorted cells with the RNeasy Mini kit (Qiagen) and was further treated with DNase I to eliminate minor DNA contaminations. Complementary DNA synthesis was done with SuperScript RT II (Invitrogen) and a mixture of oligo(dT) and random hexamer primers. SYBR Green-based real-time PCR was done with SYBR Green PCR Master Mix reagents (PerkinElmer) and an ABI PRISM 7500 (PerkinElmer) was used for the PCR. Primers for Ubb1 (ubiquitin) and mouse Card 9 were designed $^{2}$ (Supplementary Methods). The threshold cycle $\left(\mathrm{C}_{\mathrm{T}}\right)$ value for each gene of interest was normalized to that of the 'housekeeping' gene Ubbl with the equation '1.8 $8^{\text {(Ubb1-GENE) }} \times(1,000,000)$ ' as described ${ }^{32}$, where $U b b 1$ is the mean $\mathrm{C}_{\mathrm{T}}$ of triplicate ubquitin PCR, GENE is the mean $\mathrm{C}_{\mathrm{T}}$ of triplicate PCR of the gene of interest, and 1,000,000 was arbitrarily chosen as a factor to bring all values above 0 .

Generation of $\mathrm{Card9}^{-1-}$ mice. Card9 genomic DNA was obtained by PCR with genomic DNA from mouse embryonic stem cells. The targeting vector was constructed by replacement of the entire exon 1, which includes the ATG start codon and encodes a partial CARD motif, with a neomycin-resistance gene cassette driven by the promoter of the gene encoding phosphoglycerate kinase. The gene encoding herpes simplex virus thymidine kinase was inserted into the targeting vector for negative selection. After the targeting vector was transfected into embryonic stem cells, colonies doubly resistant for G418 and gancyclovir were selected and were screened by Southern blot analysis. Homologous recombinants were microinjected into C57BL/6 female mice, and heterozygous F1 progeny were intercrossed to obtain $\mathrm{Card}^{-1-}$ mice. Wild-type and $\mathrm{Card9^{-1 }}$ mice were on a 129/Sv $\times$ C57BL/6 background and were maintained according to guidelines of the Institutional Animal Care and Use Committee of the M.D. Anderson Cancer Center.

L. monocytogenes infection. Wild-type L. moncytogenes strain 10403 s was grown at $37{ }^{\circ} \mathrm{C}$ to mid-log phase (absorbance at $600 \mathrm{~nm}, 0.1$ ) and then was diluted in $500 \mu \mathrm{l}$ saline and injected intravenously into mice. The number of live bacteria lysates of infected spleens and livers was determined as described ${ }^{33}$. thole spleens and livers of wild-type and CARD9-deficient mice were collected and homogenized at various days after infection. Homogenates were plated on brain-heart infusion agar, and bacterial colony-forming units were assessed after overnight growth at $37{ }^{\circ} \mathrm{C}$. All animal studies were approved by the appropriate Institutional Animal Care and Utilization Committee.

Primary cell culture and innate immunity assays. Two to three littermate mice were used per ex vivo experiment, on average. Peritoneal macrophages were elicited by intraperitoneal injection of $4 \%$ (weight/volume) thioglycolate broth. At $4 \mathrm{~d}$ after injection, peritoneal lavage was done on mice that had been killed to obtain peritoneal macrophages, which were maintained in complete RPMI 1640 medium with $10 \%$ (volume/volume) fetal calf serum and $1 \%$ (volume/volume) penicillin-streptomycin. Bone marrow-derived macrophages were obtained from both mouse tibia and femur bones and were maintained in DMEM medium with 30\% (volume/volume) granulocyte colony-stimulating factor (produced by L929 cells), 20\% (volume/volume) fetal calf serum, 1\% (volume/volume) penicillin-streptomycin and $55 \mu \mathrm{M}$ $\beta$-mercaptoethanol. Primary macrophages were isolated and were treated for $24 \mathrm{~h}$ with PGN from S. aureus (Fluka), loxoribine, pure LPS, MDP (Invivogen), poly(I:C) and LPS (Amersham Pharmacia Biotech). IL-6 and TNF in the culture media were measured by enzyme-linked immunosorbent assay (ELISA). For bacterial infection, L. monocytogenes was added at various multiplicities of infection. After incubation for $30 \mathrm{~min}$, extracellular bacteria were removed by washing of the cells three times with PBS. For prevention of reinfection, cells were cultured in medium containing gentamycin sulfate ( $50 \mu \mathrm{g} / \mathrm{ml}$; Invitrogen). After $24 \mathrm{~h}$ of incubation, conditioned media were removed and were used for measurement of cytokines by ELISA. Vesicular stomatitis virus (Indiana strain) was added to macrophages at various multiplicities of infection for $24 \mathrm{~h}$ of incubation.

Electrophoretic mobility-shift assay (EMSA). Primary macrophages were stimulated with various inducers and nuclear extracts were prepared after the stimulation. Nuclear extracts $(5-10 \mu \mathrm{g})$ were incubated for $15 \mathrm{~min}$ at $25{ }^{\circ} \mathrm{C}$ with ${ }^{32}$ P-labeled NF- $\kappa B$ probe or transcription factor Oct-1 probe (Promega). Mixtures were then separated by $6 \%$ PAGE and viewed by autoradiography.

Note: Supplementary information is available on the Nature Immunology website.

\section{ACKNOWLEDGMENTS}

We thank K. Schluns (M.D. Anderson Cancer Center) for reagents, and G. Nunez (University of Michigan) and G. Cheng (University of California at Los Angeles) for hemagglutinin-tagged Nod2, Myc-tagged RICK and Flag-tagged RICK. Supported by the National Institutes of Health, the Leukemia and Lymphoma Society (X.L.), the Cancer Research Institute (X.L.) and the MD Anderson Cancer Center (Y.L.).

\section{AUTHOR CONTRIBUTIONS}

Y.-M.S.H., Y.Z., Y.Y., D.W., H.L. and O.D. performed the experiments. X.-F.Q., C.D. and X.L. analyzed the data.

\section{COMPETING INTERESTS STATEMENT}

The authors declare that they have no competing financial interests.

Published online at http://www.nature.com/natureimmunology/

Reprints and permissions information is available online at http://npg.nature.com/ reprintsandpermissions/

1. Akira, S., Uematsu, S. \& Takeuchi, O. Pathogen recognition and innate immunity. Cell 124, 783-801 (2006).

2. Medzhitov, R. Toll-like receptors and innate immunity. Nat. Rev. Immunol. 1, 135-145 (2001).

3. Akira, S. \& Takeda, K. Toll-like receptor signalling. Nat. Rev. Immunol. 4, 499-511 (2004).

4. Yamamoto, M. et al. TRAM is specifically involved in the Toll-like receptor 4-mediated MyD88-independent signaling pathway. Nat. Immunol. 4, 1144-1150 (2003).

5. Kawai, T., Adachi, O., Ogawa, T., Takeda, K. \& Akira, S. Unresponsiveness of MyD88deficient mice to endotoxin. Immunity 11, 115-122 (1999).

6. Matsuguchi, T., Masuda, A., Sugimoto, K., Nagai, Y. \& Yoshikai, Y. JNK-interacting protein 3 associates with Toll-like receptor 4 and is involved in LPS-mediated JNK activation. EMBO J. 22, 4455-4464 (2003).

7. Fitzgerald, K.A. et al. LPS-TLR4 signaling to IRF-3/7 and NF- $\mathrm{KB}$ involves the toll adapters TRAM and TRIF. J. Exp. Med. 198, 1043-1055 (2003).

8. Oganesyan, G. et al. Critical role of TRAF3 in the Toll-like receptor-dependent and -independent antiviral response. Nature 439, 208-211 (2006).

9. Hacker, H. et al. Specificity in Toll-like receptor signalling through distinct effector functions of TRAF3 and TRAF6. Nature 439, 204-207 (2006).

10. Kopp, E. \& Medzhitov, R. Recognition of microbial infection by Toll-like receptors. Curr. Opin. Immunol. 15, 396-401 (2003).

11. Takeda, K., Kaisho, T. \& Akira, S. Toll-like receptors. Annu. Rev. Immunol. 21, 335-376 (2003).

12. Inohara, N., Chamaillard, M., McDonald, C. \& Nunez, G. NOD-LRR proteins: role in host-microbial interactions and inflammatory disease. Annu. Rev. Biochem. 74, 355-383 (2005)

13. Yoneyama, $M$. et al. The RNA helicase RIG-I has an essential function in double-stranded RNA-induced innate antiviral responses. Nat. Immunol. 5, 730-737 (2004).

14. Inohara, N. et al. Host recognition of bacterial muramyl dipeptide mediated through NOD2. Implications for Crohn's disease. J. Biol. Chem. 278, 5509-5512 (2003).

15. Xu, L.G. et al. VISA is an adapter protein required for virus-triggered IFN- $\beta$ signaling. Mol. Cell 19, 727-740 (2005).

16. Seth, R.B., Sun, L., Ea, C.K. \& Chen, Z.J. Identification and characterization of MAVS, a mitochondrial antiviral signaling protein that activates NF-KB and IRF 3. Cell 122 , 669-682 (2005)

17. Kawai, T. et al. IPS-1, an adaptor triggering RIG-I- and Mda5-mediated type I interferon induction. Nat. Immunol. 6, 981-988 (2005).

18. Meylan, E. et al. Cardif is an adaptor protein in the RIG-I antiviral pathway and is targeted by hepatitis C virus. Nature 437, 1167-1172 (2005).

19. Chin, A.l. et al. Involvement of receptor-interacting protein 2 in innate and adaptive immune responses. Nature 416, 190-194 (2002).

20. Kobayashi, K. et al. RICK/Rip2/CARDIAK mediates signalling for receptors of the innate and adaptive immune systems. Nature 416, 194-199 (2002).

21. Sun, Q. et al. The specific and essential role of MAVS in antiviral innate immune responses. Immunity 24, 633-642 (2006). 
22. Bertin, J. et al. CARD9 is a novel caspase recruitment domain-containing protein that interacts with BCL10/CLAP and activates NF-kB. J. Biol. Chem. 275, 41082-41086 (2000).

23. Nakamura, S. et al. Overexpression of caspase recruitment domain (CARD) membraneassociated guanylate kinase 1 (CARMA1) and CARD9 in primary gastric B-cell lymphoma. Cancer 104, 1885-1893 (2005).

24. Gross, 0 . et al. Card 9 controls a non-TLR signalling pathway for innate anti-fungal immunity. Nature 442, 651-656 (2006).

25. Wang, D. et al. A requirement for CARMA1 in TCR-induced NF-KB activation. Nat. Immunol. 3, 830-835 (2002).

26. Han, J. \& Ulevitch, R.J. Limiting inflammatory responses during activation of innate immunity. Nat. Immunol. 6, 1198-1205 (2005).

응

7. Ashwell, J.D. The many paths to p38 mitogen-activated protein kinase activation in the immune system. Nat. Rev. Immunol. 6, 532-540 (2006).
28. Dong, C., Davis, R.J. \& Flavell, R.A. MAP kinases in the immune response. Annu. Rev. Immunol. 20, 55-72 (2002).

29. Alexopoulou, L., Holt, A.C., Medzhitov, R. \& Flavell, R.A. Recognition of doublestranded RNA and activation of NF- $\kappa$ B by Toll-like receptor 3 . Nature 413, 732-738 (2001).

30. Takeuchi, 0. et al. Differential roles of TLR2 and TLR4 in recognition of gram-negative and gram-positive bacterial cell wall components. Immunity 11, 443-451 (1999).

31. Girardin, S.E. et al. Nod2 is a general sensor of peptidoglycan through muramyl dipeptide (MDP) detection. J. Biol. Chem. 278, 8869-8872 (2003).

32. Duramad, O. et al. IL-10 regulates plasmacytoid dendritic cell response to CpG containing immunostimulatory sequences. Blood 102, 4487-4492 (2003).

33. Edelson, B. \& Unanue, E. MyD88-dependent but Toll-like receptor 2-independent innate immunity to Listeria: no role for either in macrophage listericidal activity. J. Immunol. 169, 3869-3875 (2002). 